\title{
Measurement of Water Level of Drum of Thermal Generator Set
}

\author{
Xian Zhang \\ School of automation, Wuhan University of Technology, Wuhan 430063, China
}

\begin{abstract}
Keywords: Water Level of Drum; Water level measurement.
\end{abstract}
\begin{abstract}
During operation of the thermal power plant, the water level of drum is the important monitoring parameter, involving not only the DAS system monitoring, but also input of auto and interlock protection of water supply. From the actual situation, this paper analyzes the cause and harm of inaccurate measurement of water level, and puts forward the proper installment method through analysis of measurement principle. At the same time, for problems easy to occur in installation and commissioning, this paper carries out summarization and puts forward the improvement opinion.
\end{abstract}

\section{Introduction}

Now, the operators running large thermal power plant need to monitor more and more parameters, and the requirement for automation level is higher and higher in order to reduce the operator's working pressure and improve security and reliability of the operation. As an important part of the boiler, measurement and monitoring of water level of the drum is particularly important.

The drum is highly valued as the high-temperature and high-pressure equipment. Measurement accuracy of water level of drum not only relates to the quality of steam, but also plays an important role in safe operation of the drum. The high water level of drum causes water entrained by steam, to deteriorate the quality of steam, resulting in deposit of superheated and steam turbine components, decrease in thermal efficiency, and water impact of steam turbine in serious case; too low water level damages the boiler's water cycle and causes over temperature of the boiler, which will cause damages to the drum in serious case. Since the medium in the drum is the high-temperature and high-pressure equipment, the boiler's sudden combustion condition or sudden change in unit load causes the pressure changes inside the drum, increasing or decreasing the bubble content in water volume, to cause volume expansion or contraction of media and result in increases or decreases in water level of drum, which will cause the false water level. Therefore, in order to improve the measurement accuracy of water level of drum, people have tried many ways, to overcome the "false water level".

\section{Measurement of Water Level of Drum}

Measurement Principle for Water Level of Drum. At present, the common measurement method of water level is local two-color mica water level gauge, electric contact water level gauge and differential pressure water level gauge. Twenty-five Major Requirements for Prevention of Major Accidents in Power Production stipulates that the protection of water level measurement is using differential pressure water level gauge. The measurement principle of water level of drum is introduced below.

Differential pressure water level gauge is to measure the water level of drum by using the principle of comparing difference in height of water column, which will compare the pressure generated by water column corresponding to water level of drum with the reference constant water level in balance container for the measurement, and the benchmark for comparison reference is the centerline of the sampling hole on water side of water level gauge. Since the reference height is unchanged, the measured differential pressure can directly reflect water level in the drum. The height of reference water column is the centerline of water surface in balance container to sampling hole on water side of water level gauge. After installation of balance container, the height of reference water column is fixed, and the range of differential pressure transmitter for measuring differential pressure should be equal to the height of reference water column. 
For the balance container, it generally uses single-chamber type, which is a ball shaped container. A tube mouth is led out from side of the container horizontally to connect with sampling tube on steam side on the drum, and a tube mouth is led out directly from the bottom of the container to connect with the positive pressure side of differential pressure transmitter. In this way, the saturated steam entering in the container is condensed into water continuously, and the extra condensed water flows back to the drum along the sampling tube. Therefore, for installation of sampling tube on steam side, the slope of 100:1 inclined to the drum side should be kept. The temperature of the water on the surface in the container is necessarily close to the saturation temperature, and balance container and its lower tube are cooled in such environment. Therefore, with decrease in the height, the temperature of reference water column will soon drop to near the ambient temperature. The average temperature of reference water column may be higher than ambient temperature, but much lower than the saturation temperature. The determination method of average temperature of reference water column and automatic modification of measurement of water level of drum need necessary test and calculation. At present, it is known that the average temperature of reference water column is considered based on constant when foreign manufacturers use the differential pressure level gauge to measure the water level of drum, and the constant generally used is $50^{\circ} \mathrm{C}$ or $60^{\circ} \mathrm{C}$.

Since the pressure in the drum varies with changes in pressure of whole boiler, the weight of saturated water, saturated steam in the drum will change during the boiler's start-up procedure or variable-pressure operation, resulting in different pressure generated by same water level of the drum.

\section{Regulations on Installation of Measurement Device for Water Level of Drum}

The commonly used field measurement device for water level of drum is balance container and differential pressure transmitter. The balance container has two types: single-chamber and dual-chamber balance container. For the low pressure boiler, since pressure and temperature have has little impact on the measurement results, the dual-chamber balance container is usually used. For the high parameter boiler, the single-chamber balance container is usually used, but compensation of temperature and pressure is needed. The difference of Insulation: the insulation must be used between the drum and balance container (the lead tube); dual-chamber balance container is insulated in the upper half, but not insulated in the lower half; the tube of single-chamber balance container and lower part of the container forming the reference water column should not be insulated. Two tubes led to the differential pressure transmitter should be laid parallel with common insulation.

\section{Requirements for Installation of Differential Pressure Measurement Device}

The balance container of differential pressure water level measuring device should be single-chamber balance container, which is the ball or ball-end cylinder with diameter of $100 \mathrm{~mm}$ (volume of $300-800 \mathrm{ml}$ ), and the sampling tube on steam \& water side in front of the container may be equipped with connecting tube.

For installation of sampling tube on steam \& water side, the slope of the tube should be no less than 100:1. The side of sampling hole of sampling tube on steam side should be low, and the side of sampling hole of sampling tube on water side should be high.

Sampling tube on steam \& water side, sampling valve and connecting tube should be well insulated. Two tubes led to the differential pressure transmitter should be laid parallel with common insulation, and anti-freezing measures should be taken as required.

\section{Effect of Ambient Temperature on the Measurement}

The compensation of temperature is realized through setting of density of condensed water in balance container (such value is determined by the drum pressure and temperature of condensed water in balance container), and the ambient compensation temperature used by DCS is $40^{\circ} \mathrm{C}$ or $60^{\circ} \mathrm{C}$. However, this will be advantageous that ventilation is different on both sides near the boiler drum due 
to season change; and different heat radiation of balance container on both sides will cause different ambient compensation temperature.

Solution: three remote-transmitting thermometer can be installed at lower part of the balance container, to measure the temperature of condensed water at lower part of the balance container, thus to modify the density of condensed water at any moment to achieve accurate measurement of the water level. Take mean value among three values of three thermometers to ensure accurate measurement, and the logic is shifting to the mode of mean value in two when the alarm is sent for broken circuit.

\section{Problems Easy to Occur in Operation of Water Level Transmitter of Drum}

The connections of primary and secondary gate as well as transmitter leak or weep, and loose balance gate will affect accurate measurement of water level.

That insulation of balance container is not completed according to the regulation will affect the measurement.

The verification range of transmitter should be same as the actual field measurement value, and modify the installation position of transmitter on site to cause changes in zero point.

Every differential pressure water level gauge should have independent sampling hole and tube, because one sampling hole or tube used for several water level gauges will cause mutual interference, and other water level gauges will be affected especially when the individual water level gauge washes the tube or connector is exposed, which will lose the significance of redundant equipment of water level gauges.

To avoid freezing of tube when the ambient temperature is over low, attention should be paid to insulation and anti-freezing of the tube. Two tubes led to the differential pressure transmitter from the balance container should be laid parallel with common insulation and heating. In addition, differential pressure transmitter should be installed in the insulation box, and close to the sampling as much as possible. The drum chamber should be sealed in winters for anti-freezing.

\section{Important Suggestions for Measurement of Water Level}

To measure the water level of drum correctly to ensure the safety of the boiler, it is suggested that the following problems should be concerned:

Local water level gauge cannot reflect changes in water level of drum correctly, so remote-transmitting water level gauge should not be used as standard water level to check the remote-transmitting water level gauge. After zero water level of local water level gauge is verified, zero water level of remote-transmitting water level gauge can be marked in rated conditions and normal water level.

The performance of electrical contact water level gauge is the same as local water level gauge, which should be used to provide protection signal.

The correct sampling method of differential pressure water level gauge is to directly lead the single-chamber balance container to sampling hole on steam side of the drum, and lead tube of balance container and sampling tube on water side of the drum are led to the differential pressure transmitter. The branch cannot be led from steam \& water side of sampling tube and other parts cannot be installed, and the range of differential pressure transmitter should be height of water column corresponding to distance of sampling hole on the drum.

\section{Summary}

The accuracy of water level measurement relates to safe, economical and stable operation of the boiler. Therefore, much attention should be paid during the installation phase, and every small link may affect the accuracy of water level measurement. Only paying attention to factors affecting the 
measurement at the same time of improving installment method constantly can provide accurate and reliable signal of water level measurement for the operators.

\section{Reference}

[1] Zhicheng Han, Yanfeng Zeng, Haibo Wang.Boiler technical question and answer [M]. Beijing: China Electric Power Press. 2008. p67-92.

[2] Ziliang Hou. Boiler drum water level measurement system [M]. Beijing: China Electric Power Press. 2005. p156-182. 